\title{
Continuous, noninvasive monitoring of local microscopic inflammation using a genetically engineered cell-based biosensor
}

\author{
Yiman Meng ${ }^{1,2}$, Ayumi Kasai ${ }^{1}$, Nobuhiko Hiramatsu ${ }^{1}$, Kunihiro Hayakawa ${ }^{1}$, \\ Kozue Yamauchi ${ }^{1,3}$, Masayuki Takeda ${ }^{4}$, Hiroshi Kawachi ${ }^{5}$, Fujio Shimizu ${ }^{5}$, \\ Jian Yao $^{1}$ and Masanori Kitamura ${ }^{1}$ \\ ${ }^{1}$ Department of Molecular Signaling, Interdisciplinary Graduate School of Medicine and Engineering, \\ University of Yamanashi, Yamanashi, Japan; ${ }^{2}$ Organ Transplantation Unit, 1st Affiliated Hospital, China \\ Medical University, Shenyang, China; ${ }^{3}$ Department of Medicine, Interdisciplinary Graduate School of \\ Medicine and Engineering, University of Yamanashi, Yamanashi, Japan; ${ }^{4}$ Department of Urology, \\ Interdisciplinary Graduate School of Medicine and Engineering, University of Yamanashi, Yamanashi, \\ Japan and ${ }^{5}$ Department of Cell Biology, Institute of Nephrology, Niigata University Graduate School \\ of Medical and Dental Sciences, Niigata, Japan
}

\begin{abstract}
Using an inflammation-responsive regulatory element as a molecular sensor, we established a cell-based biosensor for continuous, noninvasive monitoring of local microscopic inflammation in vivo. Glomerular mesangial cells were stably transfected with a marker gene encoding secreted alkaline phosphatase (SEAP) under the control of the $\kappa \mathrm{B}$ enhancer elements. The established cells secreted SEAP in vitro in response to proinflammatory cytokines as well as to soluble factors produced by inflamed glomeruli. To examine feasibility of using the established cells for in vivo monitoring of local microscopic inflammation, the sensor cells were transferred selectively into rat glomeruli via the renal circulation. After induction of acute glomerulonephritis, the serum level of SEAP was increased transiently in cell-transferred nephritic rats. The kinetics of serum SEAP was closely correlated with the natural course of the inflammation, and the increase in SEAP was attenuated by suppression of inflammation using an immunosuppressive drug, cyclophosphamide. Neither cell-transferred normal rats nor nephritic rats without cell transfer exhibited increase in the serum level of SEAP. When the sensor cells were transferred extrarenally, elevation of serum SEAP was not observed in nephritic rats, confirming that the locally settled sensor cells responded only to local inflammation. These results suggested that, without invasive procedures like tissue biopsies, continuous monitoring of microscopic inflammation is feasible in vivo via locally created, cell-based biosensors.
\end{abstract}

Laboratory Investigation (2005) 85, 1429-1439. doi:10.1038/labinvest.3700341; published online 29 August 2005

Keywords: inflammation; glomerulonephritis; biosensor; secreted alkaline phosphatase; nuclear factor- $\kappa \mathrm{B}$

For the treatment of glomerulonephritis, timing and duration of drug administration must be based on precise assessment of the local microscopic inflammation. Although serological and urinary markers are often used for this purpose, these parameters do not reflect activity of inflammation directly. Acutephase proteins including C-reactive protein are not useful markers for monitoring of glomerular

Correspondence: Dr M Kitamura, MD, PhD, Department of Molecular Signaling, Interdisciplinary Graduate School of Medicine and Engineering, University of Yamanashi, Shimokato 1110, Tamaho, Yamanashi 409-3898, Japan.

E-mail: masanori@yamanashi.ac.jp

Received 18 May 2005; revised 16 July 2005; accepted 19 July 2005; published online 29 August 2005 inflammation. The current, most reliable method for evaluation of glomerulonephritis is histopathological analysis using renal biopsy. However, tissue biopsy is invasive with a risk of complications such as massive bleeding. Furthermore, it does not allow for successive evaluation that is often necessary in unstable, fluctuating glomerulonephritis. Currently, technologies are not available for continuous, noninvasive assessment of glomerular inflammation. In this report, we aimed at creating an in vivo genetic biosensor suitable for this purpose.

We previously reported an ex vivo gene transfer approach that specifically targets the glomerulus. ${ }^{1}$ This method utilized the glomerular mesangial cell as a vector for gene delivery. That is, mesangial cells 
were cultured from isolated glomeruli, stably transfected with foreign genes in vitro and transferred back into glomeruli via the renal circulation. The injected cells distributed throughout the injected kidney and accumulated selectively in glomeruli. Sustained cell survival and transgene expression were maintained for at least several weeks. To create a local biosensor that perceives and reports the activity of glomerular inflammation, we used this cell transfer approach.

Under inflammatory situations, expression of various genes is induced in mesangial cells. For example, $\alpha$-smooth muscle actin is normally undetectable but induced in mesangial cells under various pathological conditions. ${ }^{2}$ Our previous data suggested that this induction was mediated by activation of ternary complex factors and their binding to the serum response element (SRE). ${ }^{3}$ By combining the ex vivo gene transfer method with SRE, we established a local biosensor that achieves automatic sensing of glomerular inflammation and subsequent expression of a marker gene, $\beta$-galactosidase. ${ }^{4}$ As a monitoring system for glomerular inflammation, however, this system has obvious limitations. One limitation is that $\beta$-galactosidase is not a secreted protein, and tissue biopsy is inevitable to evaluate the reporter expression. To overcome this problem, we attempted to use secreted alkaline phosphatase (SEAP) in the present report. In SEAP-transfected cells, secretion of SEAP is directly proportional to changes in intracellular SEAP mRNA and protein. ${ }^{5,6}$ This property allows SEAP to serve as a quantitative reporter for gene expression. SEAP has several important advantages over other reporter proteins. First, because sampling of cells and tissues is not required, it is possible to monitor activity of certain promoter/enhancer elements continuously. Second, SEAP activity can be measured sensitively using a conventional chemiluminescent assay. ${ }^{7}$ Third, unlike other endogenous alkaline phosphatases, SEAP is heat-stable and resistant to L-homoarginine. ${ }^{6}$ Treatment of samples with heating at $65^{\circ} \mathrm{C}$ and adding L-homoarginine eliminates influence of endogenous alkaline phosphatases. Fourth, in contrast to secreted luciferase, activity of SEAP is not affected by serum. ${ }^{8}$ When recombinant SEAP or SEAP-producing cells are injected into rats, substantial levels of SEAP can be detected in serum. ${ }^{8}$ These properties led us to use SEAP as an in vivo reporter molecule.

As we mentioned above, SRE can serve as a molecular sensor for glomerulonephritis. ${ }^{4}$ However, the promoter activity of SRE to drive foreign gene expression is relatively weak in mesangial cells. In this report, we therefore utilized an alternative, inflammation-responsive element, the $\kappa \mathrm{B}$ enhancer element, as a sensor for glomerulonephritis. Nuclear factor- $\kappa \mathrm{B}(\mathrm{NF}-\kappa \mathrm{B})$ is a ubiquitous, pleiotropic transcription factor that is involved in a variety of inflammatory processes. ${ }^{9,10}$ During the course of experimental and human glomerulonephritis, acti- vation of NF- $\kappa$ B occurs, ${ }^{11-13}$ leading to the expression of inflammation-associated genes in glomerular cells. ${ }^{14,15}$ The $\kappa$ B enhancer element should be useful as a sensor for glomerulonephritis.

In the present report, we provide experimental evidence that local inflammation can be monitored noninvasively via a locally created biosensor. Using glomerulonephritis as a model of disease, we demonstrate a feasibility of using the $\kappa \mathrm{B}$-SEAPbased sensing system for continuous, in vivo monitoring of local microscopic inflammation.

\section{Materials and methods}

\section{Cells and Reagents}

Mesangial cells (SM43) established from isolated glomeruli of a male Sprague-Dawley rat $^{1}$ were maintained in medium containing $5 \%$ fetal bovine serum (FBS). Medium containing 1\% FBS was generally used for studies. Cyclophosphamide monohydrate was purchased from Wako (Osaka, Japan). Human recombinant IL- $1 \beta$ and TNF- $\alpha$ were generous gifts of Otsuka Pharmaceutical Co. Ltd (Tokushima, Japan) and Dr Katsuo Noguchi (Teikyo University School of Medicine, Tokyo, Japan), respectively.

\section{Stable Transfection}

The sensor mesangial cell clone SM/NF- $\kappa$ B-SEAP5 was established by cotransfection of SM43 cells with pNF- $\kappa$ B-SEAP (BD Biosciences, Palo Alto, CA, USA) and pcDNA3.1. (Invitrogen, Carlsbad, CA, USA), as described previously. ${ }^{16}$ pNF- $\kappa$ B-SEAP encodes SEAP under the control of four copies of the $\kappa \mathrm{B}$ enhancer element. Mock-transfected mesangial cells expressing neo alone were established by transfection with pcDNA3.1. The mesangial cell phenotype of the established cells was confirmed by positive staining for $\alpha$-smooth muscle actin and Thy 1.1 using an anti- $\alpha$-smooth muscle actin monoclonal antibody (1:300 dilution; Sigma, St Louis, MO, USA) and an anti-Thy 1.1 monoclonal antibody 1-22-3 $(5 \mu \mathrm{g} / \mathrm{ml}),{ }^{17}$ respectively.

\section{Northern Blot Analysis}

Total RNA was extracted by a single-step method, ${ }^{18}$ and Northern blot analysis was performed as described before. ${ }^{19}$ As a SEAP probe, a $1.5 \mathrm{~kb}$ HindIII-XbaI fragment from pSEAP2-control (BD Biosciences) was radiolabeled and used for hybridization. The expression of glyceraldehyde-3-phosphate dehydrogenase was used as a loading control.

\section{SEAP Assay}

Activity of SEAP was evaluated by a chemiluminescent method using Great EscAPe SEAP detection kit 
(BD Biosciences). ${ }^{16}$ In brief, $5 \mu$ l of culture media or rat sera were mixed with $15 \mu \mathrm{l}$ of $1 \times$ dilution buffer and incubated at $65^{\circ} \mathrm{C}$ for $30 \mathrm{~min}$. After the incubation, the samples were mixed with $20 \mu$ l of assay buffer containing L-homoarginine, left at room temperature for $5 \mathrm{~min}$ and then added with $20 \mu \mathrm{l}$ of chemiluminescent enhancer containing $1.25 \mathrm{mM}$ CSPD chemiluminescent substrate. After incubation in dark for $30 \mathrm{~min}$, the samples were subjected to measurement of SEAP activity using a luminometer (Gene Light 55; Microtech Nition, Funabashi, Chiba, Japan).

\section{Crossfeeding Study}

Conditioned media were prepared from isolated normal and nephritic rat glomeruli. ${ }^{20}$ To prepare the latter, anti-Thy 1 glomerulonephritis was induced by a monoclonal antibody $1-22-3^{17}$ in adult male Sprague-Dawley rats, as described previously. ${ }^{21}$ At 4 days after the antibody injection, glomeruli were isolated, and $1 \times 10^{4}$ glomeruli were incubated for $24 \mathrm{~h}$ in $1 \mathrm{ml}$ of culture medium containing 1\% FBS. The conditioned media were collected, centrifuged to remove insoluble components and used for crossfeeding studies. In brief, sensor cells were exposed to $1: 1$ diluted $(50 \%)$ conditioned media for $24 \mathrm{~h}$, and the culture media were harvested for SEAP assay.

\section{Ex Vivo Sensing of Glomerulonephritis}

Sensor cells $\left(1 \times 10^{6}\right)$ were trypsinized and injected into the left renal artery of normal rats or nephritic rats subjected to anti-Thy 1 glomerulonephritis (day 1), as described previously. ${ }^{1,4,21}$ After the cell injection, glomeruli were isolated from both kidneys by using the conventional sieving method. ${ }^{22}$ In all, 1000 glomeruli were incubated in $100 \mu \mathrm{l}$ medium containing $1 \% \mathrm{FBS}$, and after $12 \mathrm{~h}$, media were collected for SEAP assay. To examine a possible, direct effect of the anti-Thy 1 antibody on the secretion of SEAP in sensor cells, the cells were treated with $1-22-3(0.5-5 \mu \mathrm{g} / \mathrm{ml})$ for $24 \mathrm{~h}$, and activity of SEAP was evaluated.

\section{Assessment of Cell Transfer Efficiency}

Efficiency of sensor cell transfer into glomeruli was evaluated as described before. ${ }^{23}$ In brief, trypsinized cells $\left(1 \times 10^{6}\right)$ were stained with a fluorescent dye 1,1'-dioctadecyl 3,3,3',3'-tetramethylindocarbocyanine perchlorate (DiI; Molecular Probes, Eugene, $\mathrm{OR}$, USA; $4 \mu \mathrm{g} / \mathrm{ml}$ ) at $37^{\circ} \mathrm{C}$ for $30 \mathrm{~min}$ and injected into the left renal artery of rats. After renal circulation was re-established, both kidneys were removed and used for preparation of isolated glomeruli. Isolated glomeruli were subjected to fluorescence microscopy to evaluate percentages of DiI-positive glomeruli.

\section{In Vivo Monitoring of Glomerulonephritis}

Rats were divided into four groups: normal rats, normal rats injected with sensor cells, nephritic rats and nephritic rats injected with sensor cells. In the sensor cell-injected groups, $1 \times 10^{6} \mathrm{SM} / \mathrm{NF}-\kappa \mathrm{B}-$ SEAP5 cells were injected into the left renal artery of normal rats or nephritic rats subjected to anti-Thy 1 glomerulonephritis (day 1). Sera were collected before and 1, 3, 6, 8 and 10 days after the cell injection and subjected to SEAP assay. To test whether or not the sensor cells perceive and report only the local inflammation where the sensor cells are located, nephritic rats were injected with $1 \times 10^{6}$ sensor cells intraperitoneally, and serum levels of SEAP were examined for up to 9 days. Each experimental group included at least four rats.

To confirm that injected sensor cells survived in glomeruli during the course of the study, glomeruli were isolated from both kidneys at the end of individual experiments (day 10) and cultured in the presence of $10 \%$ FBS. Outgrowing cells were selected by $330 \mu \mathrm{g} / \mathrm{ml} \mathrm{G} 418$, fixed with $1 \%$ formaldehyde and stained by hematoxylin to visualize neomycin-resistant cells.

In rats, intraperitoneal injection of immunosuppressant cyclophosphamide causes $>98 \%$ depletion of circulating leukocytes within 2 days, leading to suppression of glomerular inflammation and proteinuria in experimental glomerulonephritis. ${ }^{24}$ To examine whether therapeutic intervention in glomerulonephritis results in decrease in the level of serum SEAP, rats were given an intraperitoneal injection of cyclophosphamide $(165 \mathrm{mg} / \mathrm{kg}$ body weight) 2 days before the sensor cell injection. After 3 days (Thy 1 glomerulonephritis, day 2), sera were collected from cyclophosphamide-treated $(+)$ and -untreated (-) nephritic rats and subjected to SEAP assay.

\section{Immunofluorescence Study}

Frozen sections with $8 \mu \mathrm{m}$ thickness were prepared and incubated with an anti-rat pan macrophage/ monocyte monoclonal antibody ED1 (1:200 dilution; Chemicon International Inc., Temecula, CA, USA) for $60 \mathrm{~min}$. After washing, the sections were incubated with Alexa Fluor 488-conjugated antimouse immunoglobulins (1:1500 dilution; Molecular Probes) for $60 \mathrm{~min}$ and subjected to fluorescence microscopy. The number of ED1-positive cells per full-sized glomerulus was counted, and the mean values were used for comparison in different groups.

\section{Statistical Analysis}

Data were expressed as means \pm s.e. Statistical analysis was performed by the nonparametric Mann-Whitney $U$-test. $P<0.05$ was considered to be a statistically significant difference. Assays were performed in quadruplicate. 


\section{Results}

\section{Sensing of Proinflammatory Cytokines by Sensor Cells}

Rat mesangial cells were stably transfected with a SEAP gene under the control of the NF- $\kappa$ B enhancer elements, and an inflammation-responsive sensor clone SM/NF- $\kappa$ B-SEAP5 was established. The mesangial cell phenotype was confirmed by positive staining for $\alpha$-smooth muscle actin and Thy 1.1 (Figure 1a). To examine responsiveness of the sensor cells to proinflammatory stimuli, the cells were exposed to IL- $1 \beta$ ( $(10 \mathrm{ng} / \mathrm{ml})$ or TNF- $\alpha(250 \mathrm{U} / \mathrm{ml})$ for $24 \mathrm{~h}$. Northern blot analysis showed that the expression of SEAP mRNA was markedly induced when the cells were exposed to inflammatory cytokines (Figure 1b). Consistently, dramatic increases in the level of SEAP were observed in culture media of cytokine-exposed sensor cells $(196068 \pm 42607 \mathrm{RLU}$ in IL- $1 \beta$-exposed cells and $150865 \pm 6070 \mathrm{RLU}$ in TNF- $\alpha$-exposed cells $v s$ $2787 \pm 51$ RLU in untreated control; means \pm s.e., $P<0.05$ ) (Figure 1c). In contrast, induction of neither SEAP mRNA nor SEAP activity was observed in mock-transfected mesangial cells exposed to IL-1 $\beta$ or TNF- $\alpha$ (Figure $1 \mathrm{~d}$ and $\mathrm{e}$ ).

\section{Sensing of Glomerular Inflammation In Vitro and $E_{x}$ Vivo}

To test whether the established cells are able to perceive and report glomerular inflammation in vitro, crossfeeding studies were performed. The sensor cells were exposed for $24 \mathrm{~h}$ to media conditioned by normal rat glomeruli or nephritic glomeruli subjected to anti-Thy 1 glomerulonephritis. As shown in Figure 2, the sensor cells exposed to media conditioned by nephritic glomeruli exhibited substantial increase in SEAP secretion when compared with the cells exposed to media conditioned by normal glomeruli (20931 $\pm 245 \mathrm{RLU}$ in nephritic vs $11712 \pm 420$ RLU in normal, $P<0.05)$. Of note, modest but significant increase in the level of SEAP was also observed in the cells exposed to media conditioned by normal glomeruli.

The potential of the established cells for sensing glomerulonephritis was further examined using an technique for adoptive cell transfer., ${ }^{1,4}$ In this method, mesangial cells are transferred selectively into rat glomeruli by renal artery injection. Within glomeruli, transferred cells attach and extend along the endothelium or occupy the capillary lumen. Minor populations of transferred cells migrate into the mesangial area and extend their processes toward the surrounding glomerular capillaries. ${ }^{1}$ To evaluate the cell transfer efficiency, sensor cells were labeled with fluorescent dye DiI and injected into the left renal artery of normal rats or nephritic rats subjected to anti-Thy 1 glomerulonephritis (day 1). After the cell transfer, glomeruli were isolated from both kidneys and subjected to microscopic analysis. Figure 3a shows fluorescence microscopic analysis of glomeruli isolated from cell-injected (left) and -uninjected (right) kidneys. Accumulation of sensor cells was evident in glomeruli isolated from cell-injected left kidneys, but not in glomeruli isolated from cell-uninjected right kidneys. The percentage of DiI-positive glomeruli was $90.3 \pm 2.0 \%$ $($ mean \pm s.e.) in cell-transferred kidneys. Using these isolated glomeruli, activity of SEAP in culture media was examined after ex vivo incubation for $12 \mathrm{~h}$. As shown in Figure $3 \mathrm{~b}$, inflamed glomeruli transferred with the sensor cells exhibited substantial secretion of SEAP when compared with celltransferred, normal glomeruli (11 758 \pm 1876 RLU in nephritic vs 5074 $\pm 403 \mathrm{RLU}$ in normal, $P<0.05$ ). Consistent with the result shown in Figure 2, modest increase in the level of SEAP was also observed in normal glomeruli transferred with the sensor cells $(5074 \pm 403$ RLU in cell $(+)$ normal glomeruli vs $1040 \pm 160 \mathrm{RLU}$ in cell (-) normal glomeruli, $P<0.05)$. Neither normal nor nephritic glomeruli isolated from contralateral, uninjected kidneys showed increase in the level of SEAP.

\section{In Vivo Monitoring of Glomerulonephritis}

To evaluate usefulness of SEAP as an in vivo reporter molecule, we established mesangial cells that constitutively secrete SEAP under the control of the simian virus 40 early promoter and enhancer. When these cells were implanted into the peritoneal cavity of rats, significant increase in the level of serum SEAP was detected. ${ }^{8}$ The level of SEAP was proportional to the number of cells injected (our unpublished data).

We examined whether the established sensor cells are competent for in vivo monitoring of glomerulonephritis. For this purpose, acute anti-Thy 1

\footnotetext{
Figure 1 Sensing of proinflammatory cytokines by sensor cells. Rat mesangial cells were stably transfected with a secreted alkaline phosphatase (SEAP) gene under the control of the $\kappa \mathrm{B}$ enhancer elements, and an inflammation-responsive sensor clone SM/NF- $\kappa \mathrm{B}$ SEAP5 was established. (a) Immunofluorescence staining for mesangial cell markers, $\alpha$-smooth muscle actin and Thy 1.1. NC, negative control. (b) Northern blot analysis of SEAP expression in cytokine-exposed sensor cells. Cells were exposed to IL- $1 \beta$ ( $10 \mathrm{ng} / \mathrm{ml}$ ) or TNF- $\alpha$ $(250 \mathrm{U} / \mathrm{ml})$ for $24 \mathrm{~h}$, and expression of SEAP mRNA was evaluated. As a loading control, expression of glyceraldehyde-3-phosphate dehydrogenase (GAPHD) is shown. (c) Secretion of SEAP by sensor cells exposed to cytokines. Cells were treated with IL-1 $\beta$ or TNF- $\alpha$ for $24 \mathrm{~h}$, and activity of SEAP in culture media was evaluated by a chemiluminescent assay. Assays were performed in quadruplicate. Data are presented as means \pm s.e., and asterisks indicate statistically significant differences $(P<0.05)$. (d and e) Lack of expression and secretion of SEAP in mock-transfected mesangial cells. Cells were treated with IL-1 $\beta$ or TNF- $\alpha$ for $24 \mathrm{~h}$, and expression of SEAP mRNA (d) and activity of SEAP in culture media (e) were evaluated.
} 
glomerulonephritis was induced in rats by intravenous administration with a monoclonal antibody 1-22-3. ${ }^{17}$ Previous reports by our group and others described in detail the pathological process of acute glomerulonephritis induced by $1-22-3 .{ }^{17,25,26}$ Following the injection of 1-22-3, accumulation of macrophages is observed within $24 \mathrm{~h}$ and peaked at days $4-7 .^{25,26}$ Proteinuria is induced within $24 \mathrm{~h}$ and a
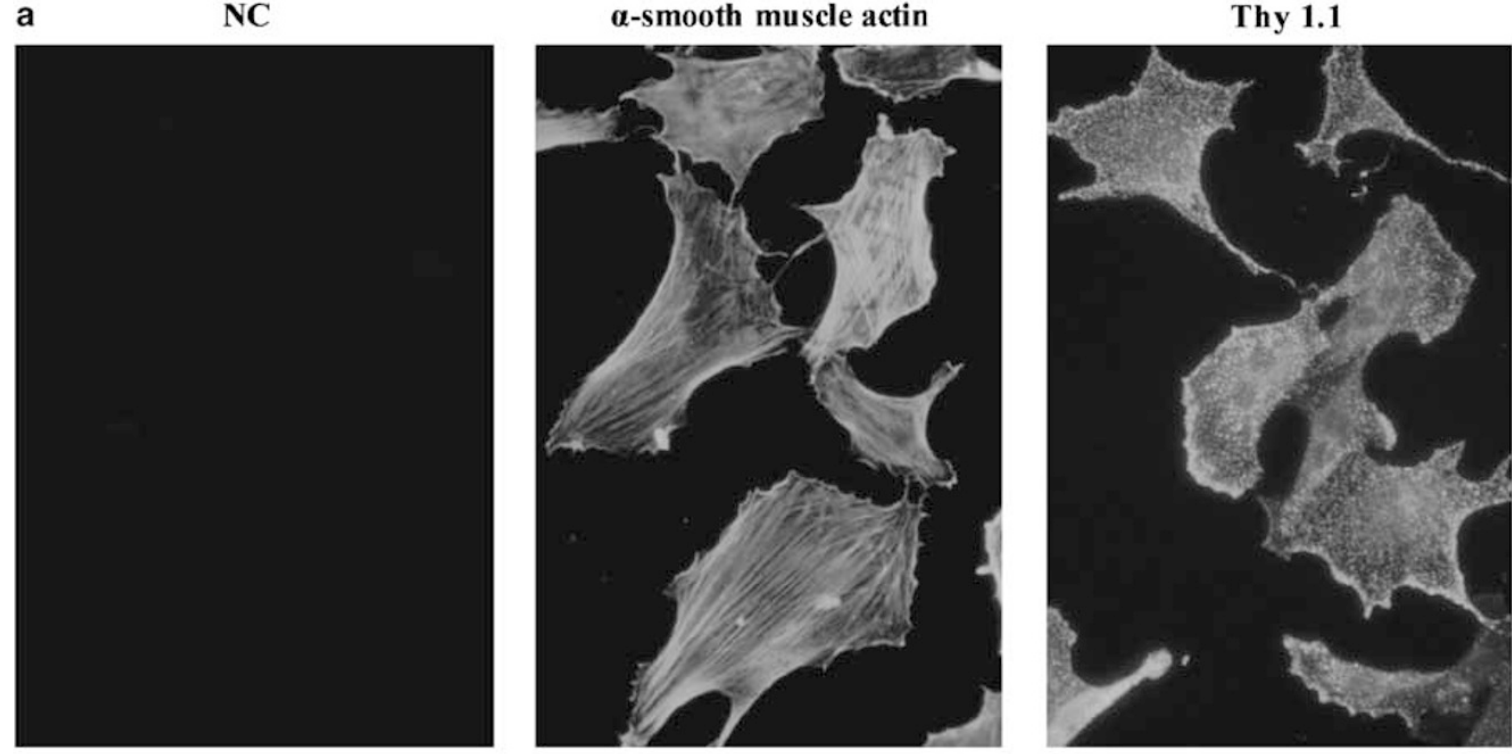

b

SEAP

GAPDH

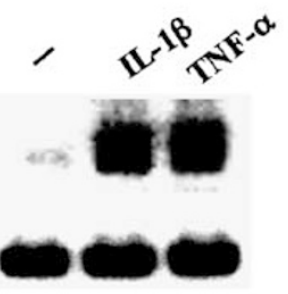

d

SEAP

GAPDH $\alpha$-smooth muscle actin
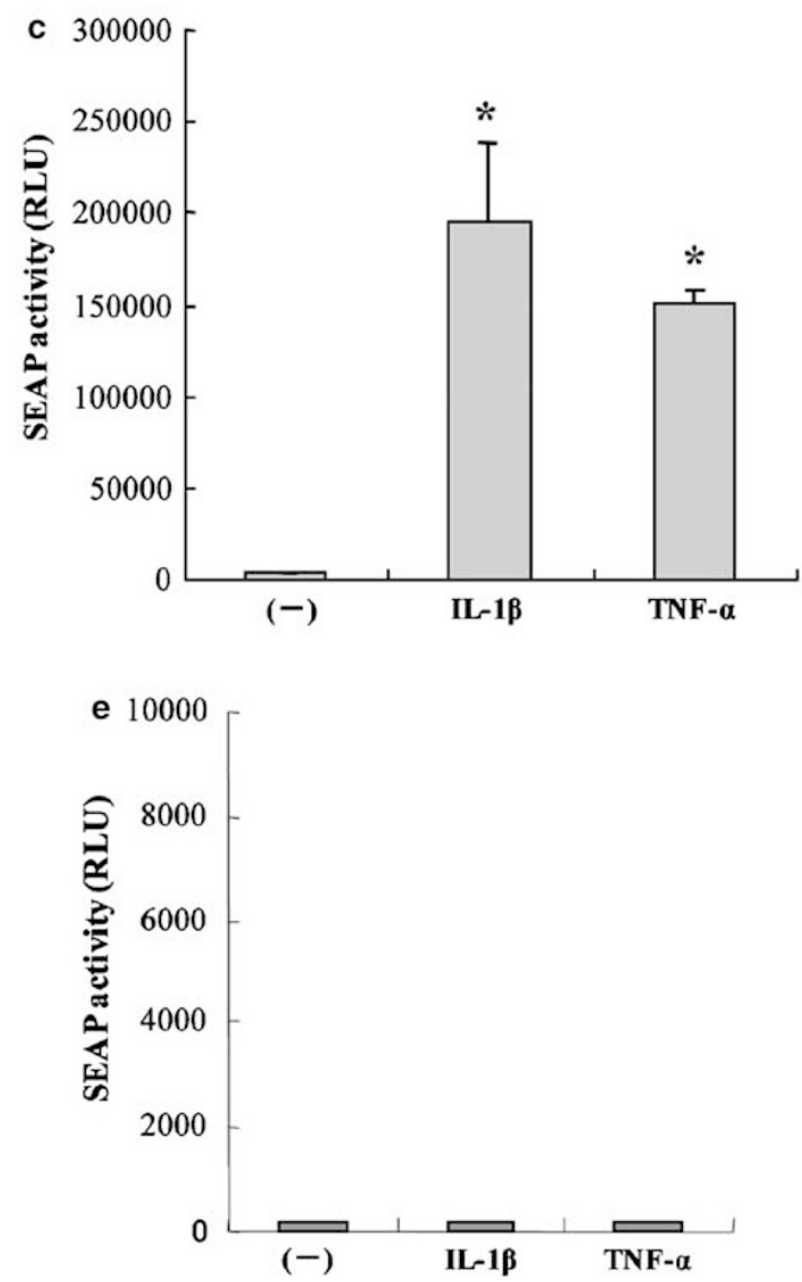


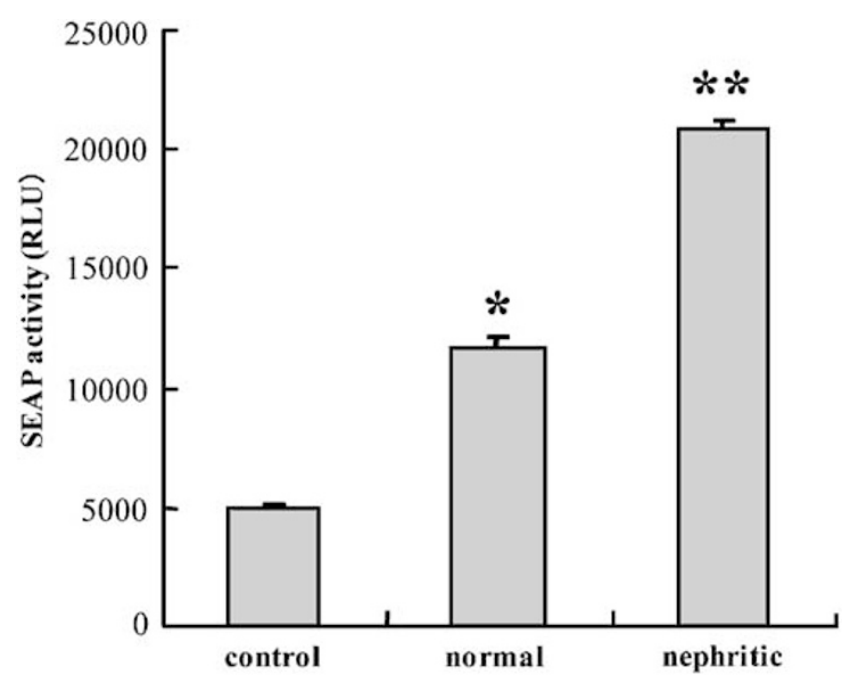

Figure 2 Sensing of glomerular inflammation in vitro by sensor cells: Crossfeeding study. Sensor cells were exposed for $24 \mathrm{~h}$ to $1: 1$ diluted $(50 \%)$ media conditioned by isolated normal or nephritic glomeruli subjected to anti-Thy 1 glomerulonephritis (day 4). The culture media were collected and subjected to SEAP assay. Assays were performed in quadruplicate. Data are presented as means \pm s.e. A single asterisk indicates a statistically significant difference against unconditioned medium (control), and a double asterisk indicates a statistically significant difference against 'normal' conditioned medium.

peaks to maximum at day $5 .{ }^{17}$ After day 7 , the inflammation shifts to the resolution phase characterized by disappearance of leukocytes, apoptosis of mesangial cells and attenuation of proteinuria. ${ }^{17,25,26}$ In the present study, we first reconfirmed the effectiveness of 1-22-3 for the induction of acute glomerulonephritis. Sensor cells were injected into the left renal artery of normal rats or nephritic rats $24 \mathrm{~h}$ following the injection of 1-22-3. After 3 days, kidneys were removed and subjected to immunohistochemical analysis of macrophages. As shown in Figure 4a, infiltration of macrophages was evident in nephritic glomeruli, but not in normal glomeruli. The number of ED1-positive macrophages was $5.20 \pm 0.75$ cells per glomerulus in nephritic kidneys against $0.50 \pm 0.24$ cells per glomerulus in normal kidneys $(P<0.05)$ (Figure 4b). This result was consistent with our previous observation. ${ }^{25}$

Based on this result, we examined feasibility of using the established sensor cells for in vivo monitoring of glomerulonephritis. For this purpose, rats were divided into four groups: normal rats (group A), normal rats injected with sensor cells (group B), nephritic rats (group C) and nephritic rats injected with sensor cells (group D). At $24 \mathrm{~h}$ after the administration with 1-22-3, the sensor cells were injected into the left renal artery, and serum was collected every 2-3 days. To examine whether localization of sensor cells at inflammatory sites is required for sensing of inflammation, cells were injected intraperitoneally into nephritic rats (group E), and serum SEAP was evaluated. In untreated normal rats, background activity of serum SEAP was $1792 \pm 281$ RLU (19 rats). After the transfer of sensor cells into glomeruli, serum levels of SEAP were increased exclusively in nephritic rats (Figure 5, group D). Significant elevation of SEAP was observed within $24 \mathrm{~h}(2.26 \pm 0.34$-fold; $P<0.05)$, peaked to maximum at days $3-6(3.46 \pm 0.44$-fold at day $3, \quad 3.47 \pm 0.85$-fold at day $6 ; \quad P<0.05$ ) and declined thereafter. This kinetics of serum SEAP was closely correlated with the natural course of inflammation in this experimental model. ${ }^{17,25,26}$ Neither untreated normal rats, cell-transferred normal rats nor untreated nephritic rats exhibited significant elevation of serum SEAP (Figure 5, groups A-C). The induction of SEAP was not due to a direct effect of 1-22-3 on sensor cells, because treatment of the cells with 1-22-3 $(0.5-5 \mu \mathrm{g} / \mathrm{ml})$ did not trigger secretion of SEAP in vitro (data not shown). When sensor cells were injected intraperitoneally in nephritic rats, elevation of SEAP activity was not observed (Figure 5, group E). This result indicated that the transferred sensor cells perceived and responded only to the local inflammation.

To confirm survival of the sensor cells in glomeruli during the course of the study, cellinjected rats were killed at day 10, and glomeruli were isolated from individual kidneys. The isolated glomeruli were cultured in the presence of $10 \%$ FBS for 1-2 weeks, and outgrowing cells were selected by G418. The sensor cells, but not intrinsic glomerular cells, express neomycin phosphotransferase and are resistant to neomycin analogue G418. Figure 6 shows a typical result of these experiments. A number of neomycin-resistant sensor cells were constantly recovered from the cultures of cellinjected, nephritic glomeruli. Similar results were also obtained in the culture of cell-injected, normal glomeruli (data not shown). In contrast, neomycinresistant cells were never recovered from normal and nephritic glomeruli isolated from contralateral right kidneys.

To examine whether the serum level of SEAP in sensor cell-transferred, nephritic rats is reduced by therapeutic intervention, we used an anti-inflammatory drug, cyclophosphamide. In rats, intraperitoneal injection of cyclophosphamide $(165 \mathrm{mg} / \mathrm{kg}$ body weight) causes substantial suppression of glomerular inflammation and proteinuria in experimental glomerulonephritis. ${ }^{20}$ Rats were given an intraperitoneal injection of cyclophosphamide 2 days before the sensor cell injection. After 3 days (Thy 1 glomerulonephritis, day 2), sera were collected from drug-treated and -untreated nephritic rats and subjected to SEAP assay. As shown in Figure 7, administration with cyclophosphamide significantly attenuated the elevation of serum SEAP in cell-transferred, nephritic rats (fold increase in serum SEAP: $1.81 \pm 0.50$-fold in cyclophosphamide-treated rats vs $3.46 \pm 0.44$-fold in cyclophosphamide-untreated rats; $P<0.05)$. This 


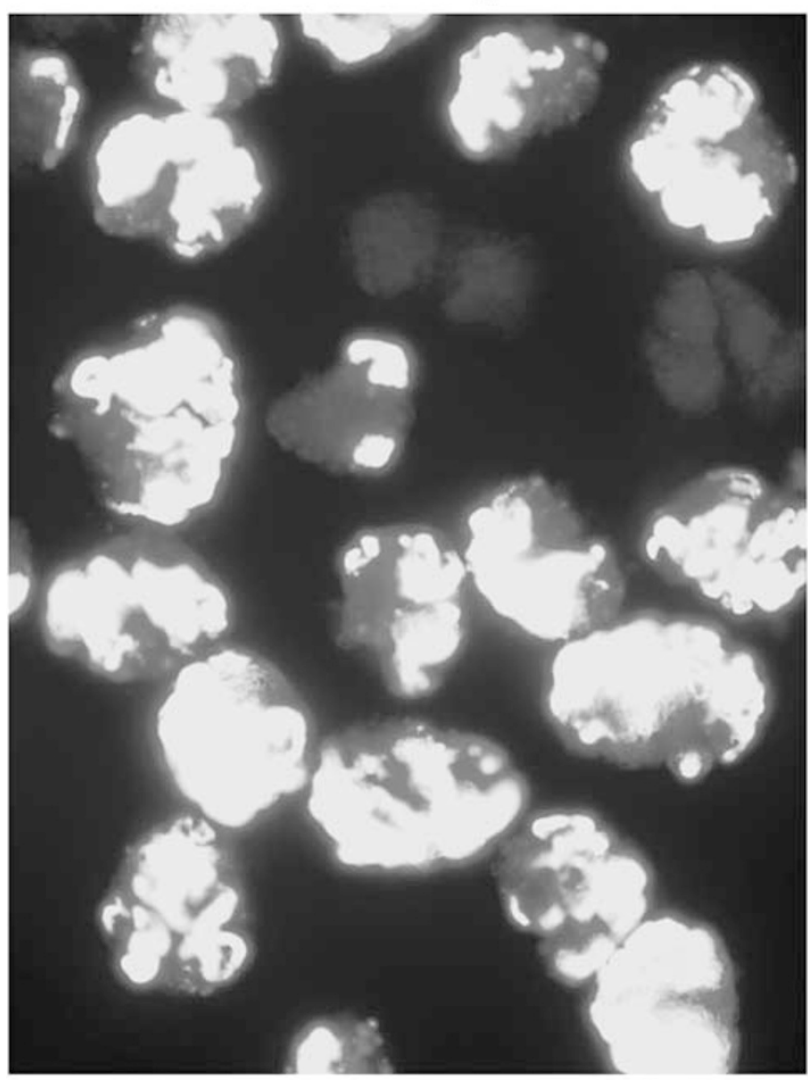

\section{Right kidney}

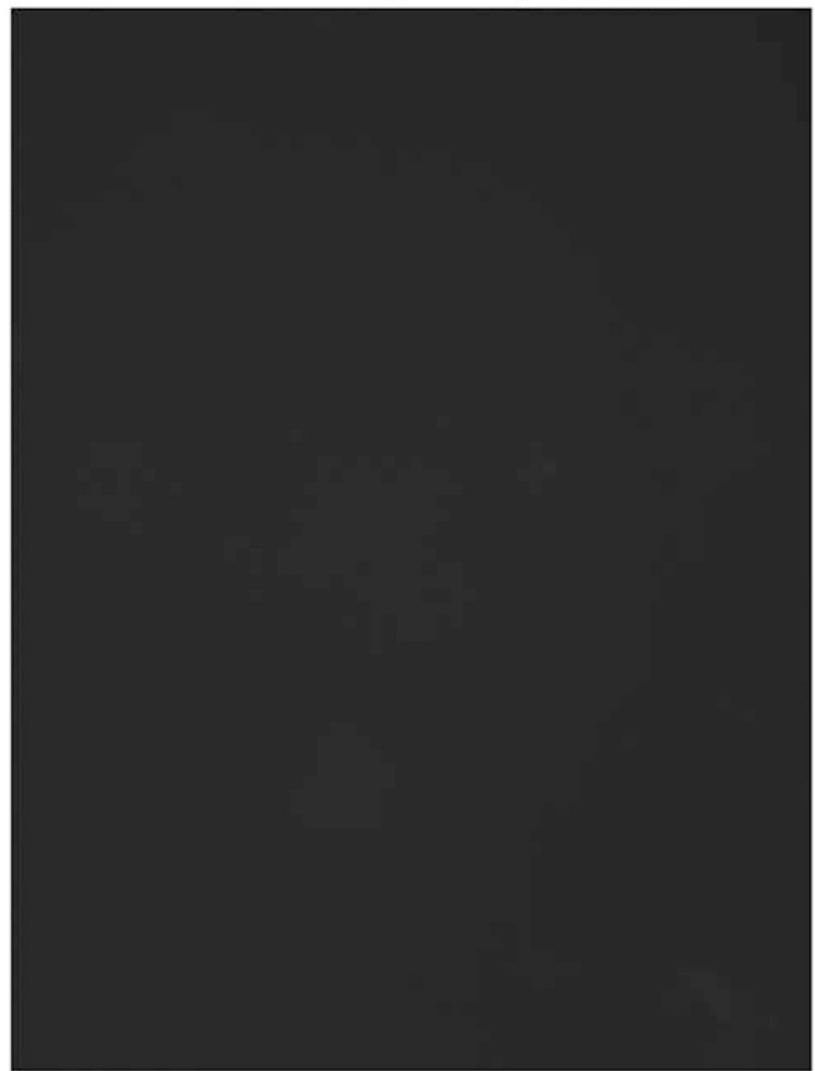

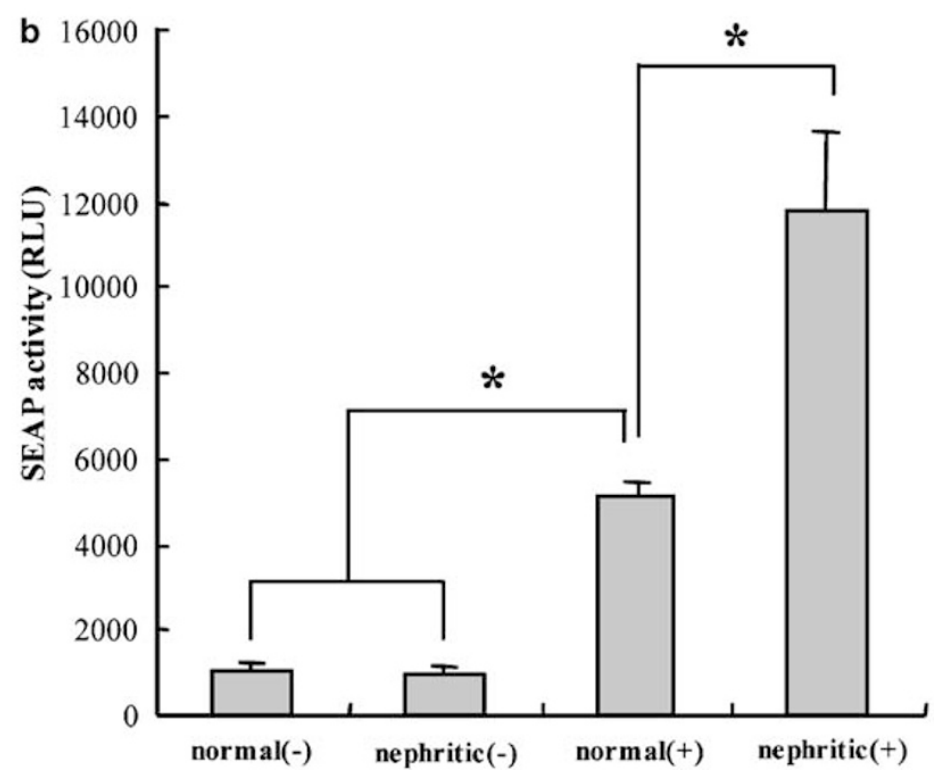

Figure 3 Sensing of glomerular inflammation in vitro by sensor cells: Cell transfer study. Sensor cells $\left(1 \times 10^{6}\right.$ cells $)$ stained with a fluorescent dye 1,1'-dioctadecyl 3,3,3',3'-tetramethylindocarbocyanine perchlorate were transferred into normal and nephritic rat glomeruli (anti-Thy 1 glomerulonephritis; day 1) via the renal circulation. After the cell injection, glomeruli were isolated from both kidneys and used for analyses. (a) Fluorescence microscopic analysis of isolated glomeruli transferred with sensor cells. Left, glomeruli isolated from cell-injected left kidneys; right, glomeruli isolated from contralateral right kidneys. (b) Levels of SEAP activity produced by isolated glomeruli. In all, 1000 glomeruli were incubated in $100 \mu \mathrm{l}$ medium containing $1 \%$ fetal bovine serum (FBS). After $12 \mathrm{~h}$, media were collected and subjected to SEAP assay. Assays were performed in triplicate. Data are presented as means \pm s.e. Asterisks indicate statistically significant differences $(P<0.05) .(+)$, glomeruli isolated from cell-injected left kidneys; $(-)$, glomeruli isolated from contralateral right kidneys without cell transfer. 
a

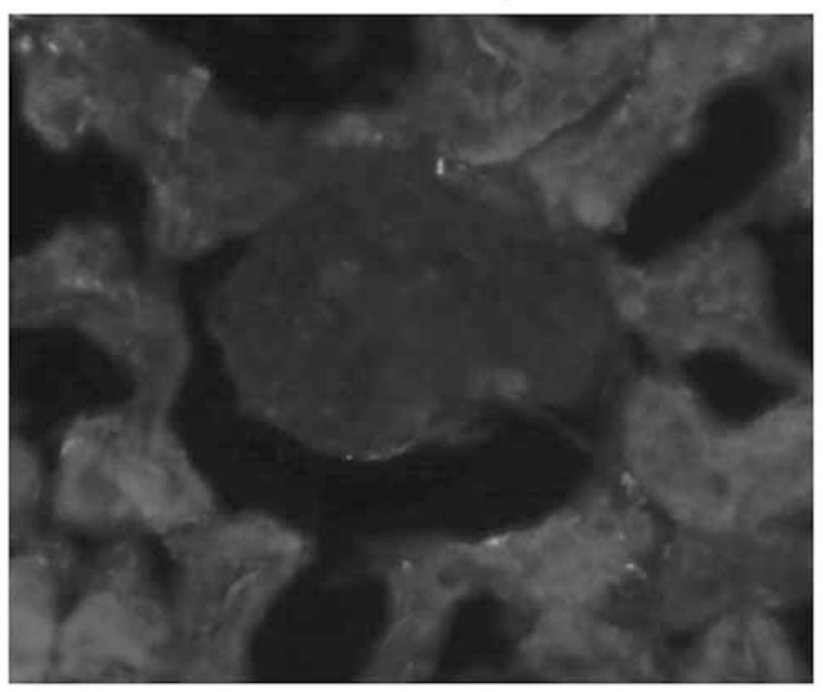

Neph ritic kidney

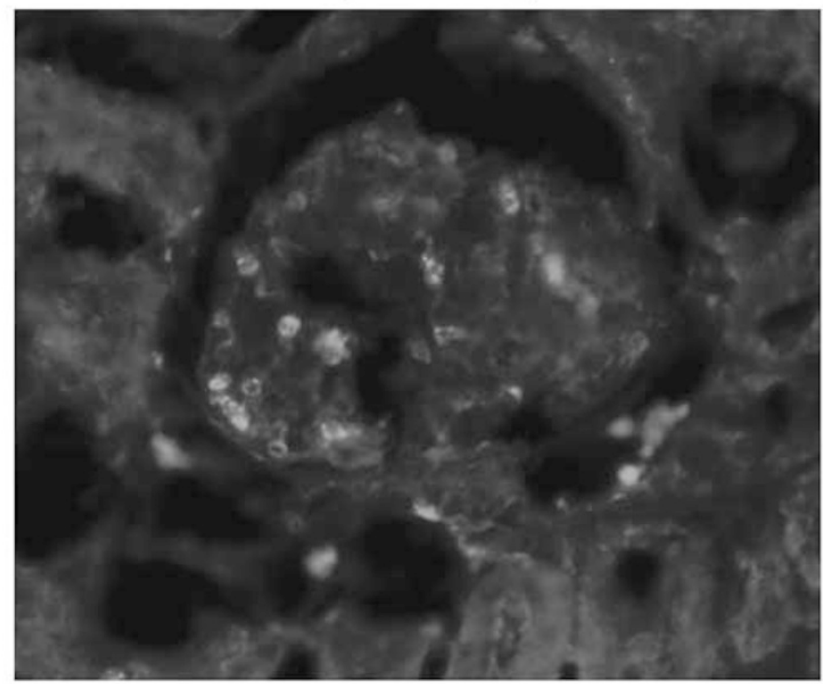

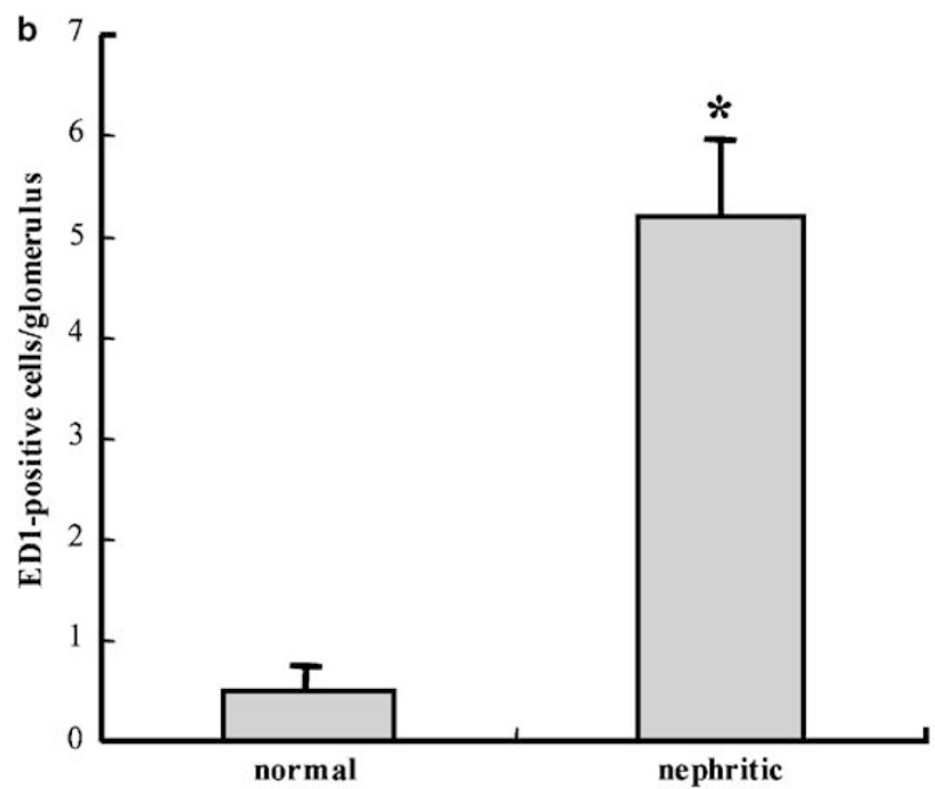

Figure 4 Accumulation of macrophages in glomeruli subjected to anti-Thy 1 glomerulonephritis. Sensor cells $\left(1 \times 10^{6}\right)$ were injected into the normal $(n=4)$ and nephritic $(n=6)$ rat kidneys subjected to anti-Thy 1 glomerulonephritis at day 1 . Before and 3 days after the cell injection, kidneys were removed and subjected to immunofluorescence analysis for accumulation of macrophages. (a) Immunofluorescence staining of macrophages in normal kidneys (left) and nephritic kidneys (right). Magnification, $\times 200$. (b) Quantitative assessment of the number of ED-1-positive monocytes/macrophages per glomerulus. Data are presented as means \pm s.e. An asterisk indicates a statistically significant difference $(P<0.05)$

result further confirmed that the usefulness of the $\kappa \mathrm{B}$-SEAP-based biosensor for monitoring of local glomerular inflammation.

\section{Discussion}

In the present report, we describe an in vivo biosensor that allows for continuous, noninvasive monitoring of local microscopic inflammation. Using the $\kappa \mathrm{B}$ enhancer element as a molecular sensor, we created sensor mesangial cells that secrete SEAP in response to inflammatory stimuli. When the established cells were transferred into inflamed glomeruli, serum levels of SEAP were significantly elevated. The kinetics of the serum SEAP was correlated with the natural course of glomerular inflammation, and its level was attenuated by therapeutic intervention.

Previous reports by our group and others described the pathological process of acute glomerulonephritis induced by the monoclonal antibody 1-22-3. ${ }^{17,25,26}$ Following the injection of 1-22-3, accumulation of leukocytes is observed within $24 \mathrm{~h}$ 
Cell (-)

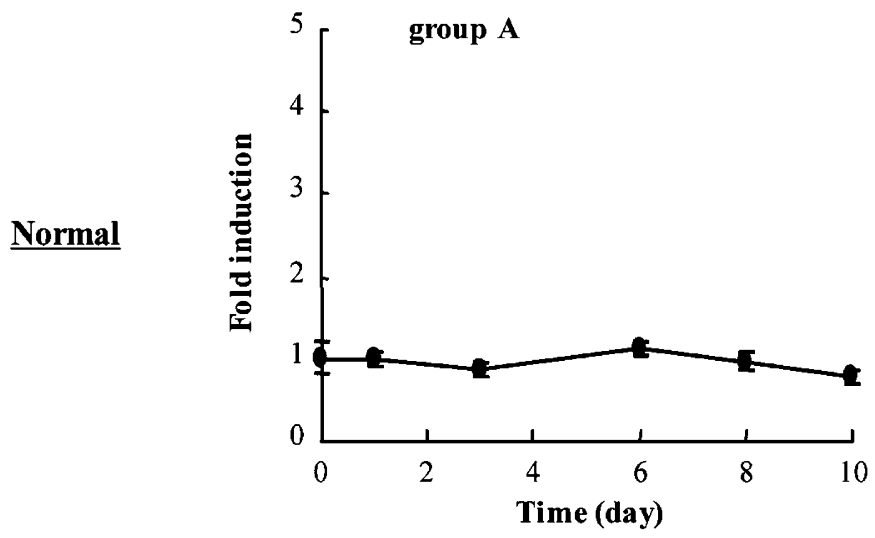

Cell (-)

Nephritic

$\underline{\text { Nephritic }}$

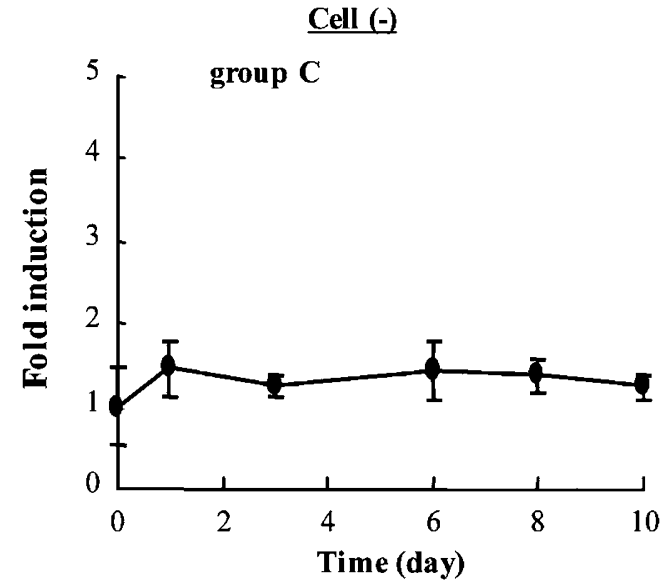

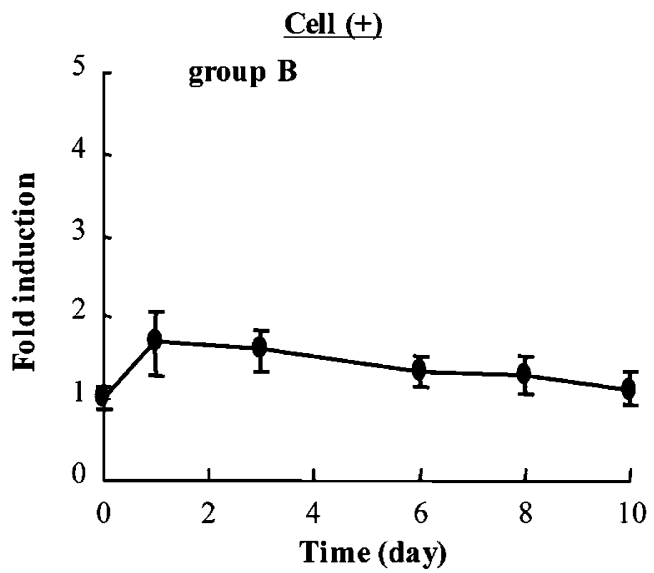

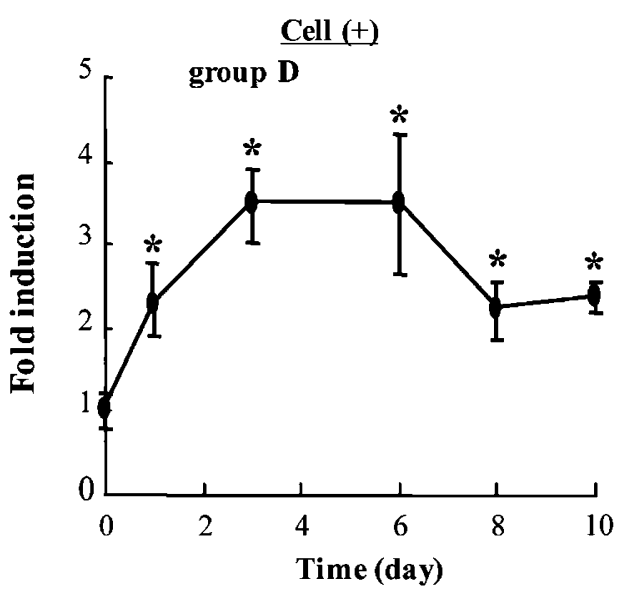

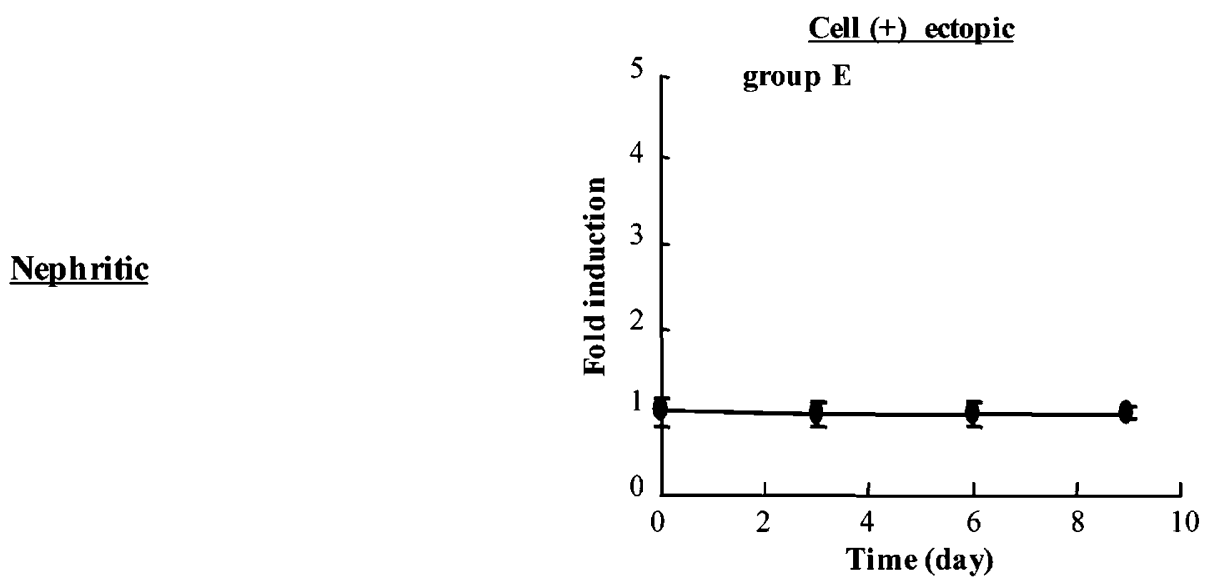

Figure 5 Successive, in vivo assessment of glomerulonephritis. Sensor cells $\left(1 \times 10^{6}\right.$ cells) were injected into the left renal artery of normal rats or nephritic rats subjected to anti-Thy 1 glomerulonephritis (day 1). Rats were divided into four groups: normal rats (group A, $n=5$ ), normal rats injected with the sensor cells (group B; $n=4$ ), nephritic rats (group C; $n=4$ ) and nephritic rats injected with the sensor cells (group D; $n=5$ ). Before and after the cell injection, sera were collected every 2-3 days and subjected to SEAP assay. To examine whether or not the sensor cells perceive and respond only to the local inflammation where the cells are located, nephritic rats were injected with $1 \times 10^{6}$ sensor cells intraperitoneally (group E; $n=4$ ), and serum SEAP levels were examined for up to 9 days. Data are presented as means \pm s.e., and asterisks indicate statistically significant differences $(P<0.05)$.

and peaks at days $4-7 .^{25,26}$ Proteinuria is induced within $24 \mathrm{~h}$ and peaks to maximum at day $5 .{ }^{17}$ After day 7 , the inflammation shifts to the resolution phase characterized by disappearance of leukocytes, apoptosis of mesangial cells and attenuation of proteinuria. ${ }^{17,25,26}$ In the present report, we demon- strated that, in parallel with this pathological process, the serum level of SEAP peaked at days 3-6 and declined thereafter in sensor cell-implanted, nephritic rats. Numerous neomycin-resistant sensor cells were constantly recovered from glomeruli even 10 days after the cell injection. This 


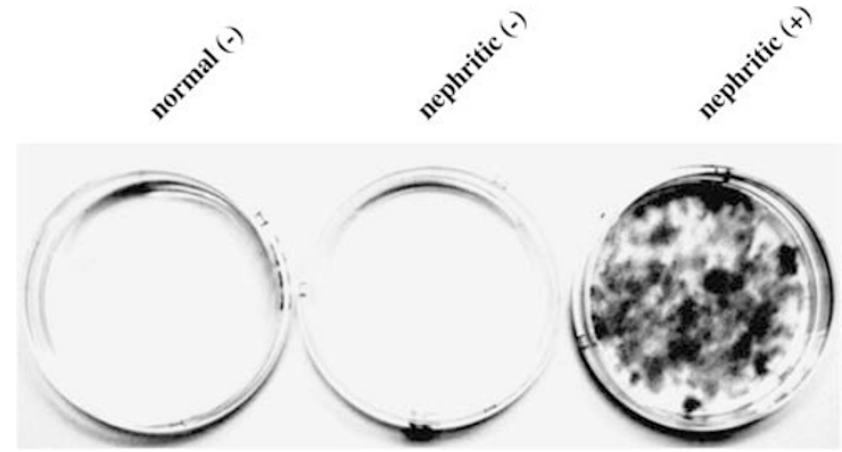

Figure 6 Recovery of sensor cells from isolated glomeruli. At the end of experiments (day 10), glomeruli were isolated from both kidneys of sensor cell-transferred, nephritic rats and cultured in the presence of $10 \%$ FBS. Nephritic $(-)$, nephritic glomeruli without sensor cells; nephritic $(+)$, nephritic glomeruli transferred with sensor cells. As a negative control, glomeruli isolated from uninjected, normal rats were used (normal $(-)$ ). Outgrowing cells were selected by G418 $(330 \mu \mathrm{g} / \mathrm{ml})$ for $1-2$ weeks, fixed with $1 \%$ formaldehyde and stained by hematoxylin to visualize neomycin-resistant sensor cells.

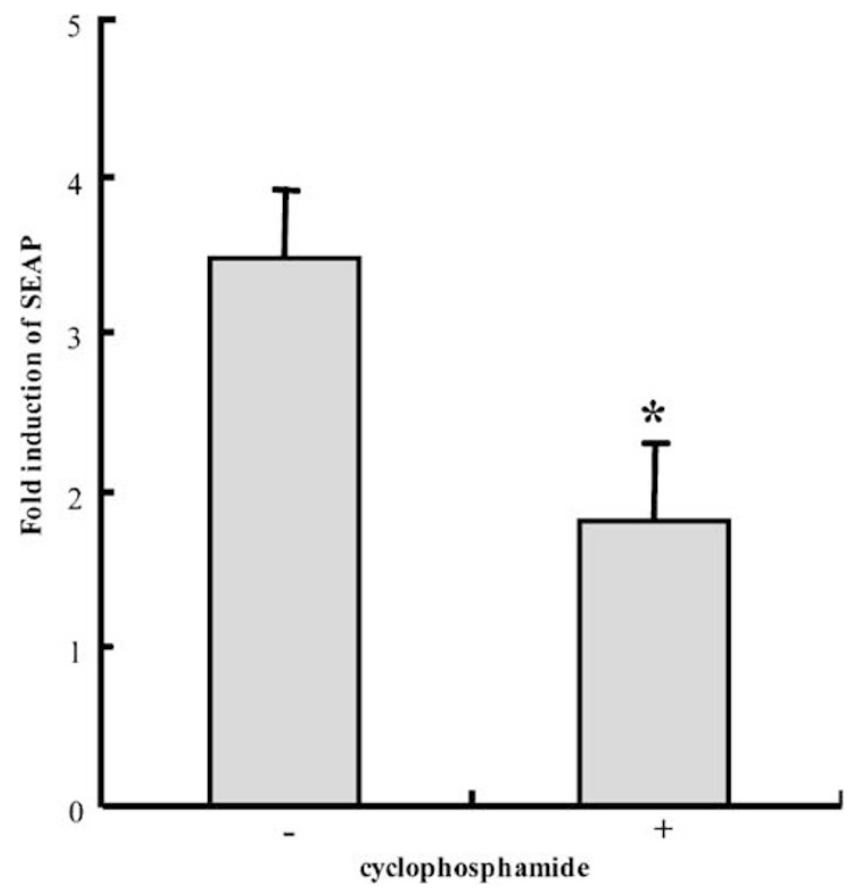

Figure 7 Monitoring of activity of glomerulonephritis treated with cyclophosphamide. Rats were given an intraperitoneal injection of cyclophosphamide ( $165 \mathrm{mg} / \mathrm{kg}$ body weight) 2 days before the sensor cell injection. After 3 days, sera were collected from cyclophosphamide-treated $(+)$ and -untreated $(-)$ nephritic rats (Thy 1 glomerulonephritis day 2), and subjected to SEAP assay. Each experimental group included four rats. Data are shown as means \pm s.e. An asterisk indicates a statistically significant difference $(P<0.05)$.

is consistent with our previous finding that genetically engineered mesangial cells transferred into rat glomeruli survive and express transgenes for at least 1 month. $^{1}$ The transferred sensor cells perceived only the local inflammation because elevation of serum SEAP was not observed in nephritic rats in which sensor cells were injected intraperitoneally. These data suggested that selective and successive assessment of glomerulonephritis is feasible in vivo by creating a local biosensor.

In this study, the $\kappa \mathrm{B}$ enhancer element was used as a sensor for glomerular inflammation. However, other sensor sequences combined with the SEAP reporter system may also be useful to monitor glomerulonephritis in vivo. Possible candidates of inflammation-responsive sequences are SRE and 12$o$-tetradecanoylphorbol-13-acetate response element (TRE). We transfected mesangial cells with expression plasmids that introduce the SEAP gene under the control of SRE or TRE. Stable clones were established and tested for the induction of SEAP in response to stimuli including serum, platelet-derived growth factor, phorbol ester and IL-1 $\beta$. In both types of sensor clones, the induction of SEAP was unexpectedly low. In contrast to the $\kappa \mathrm{B}$-based sensor cells that achieved high levels of SEAP induction up to 70 - to 80 -fold, the maximum induction rates were only two- to three-fold (our unpublished data). The low induction of SEAP in the SRE- and TRE-based sensor cells may be due to relatively high levels of basal activity of these regulatory elements and/or low inducibility in response to stimuli in cultured mesangial cells.

We used SEAP as a reporter molecule to assess glomerular inflammation. To our knowledge, this is the first to demonstrate the in vivo utility of the SEAP reporter system for evaluation of local inflammation. The activity of SEAP in serum can be measured easily, quickly and sensitively by using conventional chemiluminescent methods. This is the great advantage of the SEAP reporter system. However, one disadvantage of the in vivo SEAP reporter system is that SEAP is not excreted into urine, ${ }^{8}$ and urine-based assessment of disease activity is not possible. It is because the molecular weight of SEAP is approximately $64 \mathrm{kDa},{ }^{5}$ which is too large to be filtered through the glomerular basement membrane. Other smaller reporter molecules such as human chorionic gonadotropin could be used for urine-based monitoring of local disease activity ${ }^{27}$ but further investigation will be needed to examine this possibility.

In this report, an ex vivo gene transfer method was used to create the local biosensor in glomeruli. The advantage of this approach is that it achieves sitespecific delivery of sensor cells and sustained transgene expression. The transferred cells reside within the glomerular capillaries or the mesangium, and sustained survival and transgene expression are observed for at least 4 weeks. ${ }^{1}$ However, a limitation of this approach is that the transferred cells can proliferate and synthesize extracellular matrix in response to local inflammatory mediators and may exacerbate pathologic changes. Indeed, we found that 1-22-3-induced nephritic glomeruli transferred with mesangial cells exhibited enhanced hypercel- 
lularity and matrix accumulation. To solve this problem, use of replication-incompetent sensor cells created by genetic manipulation or by pretreatment with antimitogenic agents may be useful. ${ }^{1}$ Alternatively, direct, in vivo transfer of the $\kappa \mathrm{B}$-SEAP construct into glomerular cells using, for example, viral vectors may also be useful to overcome the problem. Compare with the ex vivo gene transfer approach, the direct, in vivo gene transfer may be more realistic for the purpose of clinical applications. Currently, several in vivo gene transfer methods targeting glomeruli are available. Although the majority of those systems achieve only transient gene expression, combination of the $\kappa \mathrm{B}-\mathrm{SEAP}$ construct with some advanced viral vectors would allow for application of this system in human.

\section{Acknowledgements}

This work was supported by Grants-in-Aid for Scientific Research from the Ministry of Education, Culture, Sports, Science and Technology, Japan (16390243 to MK).

\section{References}

1 Kitamura M, Taylor S, Unwin R, et al. Gene transfer into the rat renal glomerulus via a mesangial cell vector: site-specific delivery, in situ amplification and sustained expression of an exogenous gene in vivo. J Clin Invest 1994;94:497-505.

2 Johnson RJ, Floege J, Yoshimura A, et al. The activated mesangial cell: a glomerular 'myofibroblast'? J Am Soc Nephrol 1992;2:S190-S197.

3 Kitamura M, Ishikawa Y. Three-dimensional matrix primes mesangial cells to downregulation of $\alpha$-smooth muscle actin via deactivation of CArG box elements. Kidney Int 1998;53:690-697.

4 Kitamura M, Kawachi H. Creation of an in vivo cytosensor using engineered mesangial cells: automatic sensing of glomerular inflammation controls transgene activity. J Clin Invest 1997;100:1394-1399.

5 Berger J, Hauber J, Hauber R, et al. Secreted placental alkaline phosphatase: a powerful new quantitative indicator of gene expression in eukaryotic cells. Gene 1988;66:1-10.

6 Cullen BR, Malim MH. Secreted placental alkaline phosphatase as a eukaryotic reporter gene. Methods Enzymol 1992;216:362-368.

7 Alam J, Cook JK. Reporter genes: application to the study of mammalian gene transcription. Anal Biochem 1990;188:245-254.

8 Hiramatsu N, Kasai A, Meng Y, et al. Alkaline phosphatase vs luciferase as secreted reporter molecules in vivo. Anal Biochem 2005;339:249-256.

9 Baeuerle PA, Henkel T. Function and activation of $\mathrm{NF}-\kappa \mathrm{B}$ in the immune system. Annu Rev Immunol 1994;12:141-179.

10 Barnes PJ, Karin M. Nuclear factor $-\kappa$ B: a pivotal transcription factor in chronic inflammatory diseases. N Engl J Med 1997;336:1066-1071.
11 Sakurai H, Hisada Y, Ueno M, et al. Activation of transcription factor NF- $\kappa \mathrm{B}$ in experimental glomerulonephritis in rats. Biochim Biophys Acta 1996;1316: 132-138.

12 Ashizawa M, Miyazaki M, Abe K, et al. Detection of $\mathrm{NF}-\kappa \mathrm{B}$ in IgA nephropathy using Southwestern histochemistry. Am J Kidney Dis 2003;42:76-86.

13 Sakai N, Wada T, Furuichi K, et al. p38 MAPK phosphorylation and NF- $\kappa$ B activation in human crescentic glomerulonephritis. Nephrol Dial Transplant 2002;17:998-1004

14 Lopez-Franco O, Suzuki Y, Sanjuan G, et al. NF- $\kappa \mathrm{B}$ inhibitors as potential novel anti-inflammatory agents for the treatment of immune glomerulonephritis. Am J Pathol 2002;161:1497-1505.

15 Tomita N, Morishita R, Tomita S, et al. Inhibition of TNF- $\alpha$-induced cytokine and adhesion molecule. Expression in glomerular cells in vitro and in vivo by transcription factor decoy for NF- $\kappa$ B. Exp Nephrol 2001;9:181-190.

16 Meng Y, Kasai A, Hiramatsu N, et al. Real-time monitoring of mesangial cell-macrophage cross-talk using SEAP in vitro and ex vivo. Kidney Int 2005; 68:886-893.

17 Kawachi H, Orisaka M, Matsui K, et al. Epitopespecific induction of mesangial lesions with proteinuria by a MoAb against mesangial cell surface antigen. Clin Exp Immunol 1992;88:399-404.

18 Chomczynski P, Sacchi N. Single-step method of RNA isolation by acid guanidinium thiocyanatephenol-chloroform extraction. Anal Biochem 1987; 162:156-159.

19 Kitamura M. Identification of an inhibitor targeting macrophage production of monocyte chemoattractant protein-1 as TGF- $\beta$. J Immunol 1997;159:1404-1411.

20 Kitamura M, Sütö T, Yokoo T, et al. Transforming growth factor- $\beta$ is the predominant paracrine inhibitor of macrophage cytokine synthesis produced by glomerular mesangial cells. J Immunol 1996;156: 2964-2971.

21 Kitamura M. Creation of a reversible on/off system for site-specific in vivo control of exogenous gene activity in the renal glomerulus. Proc Natl Acad Sci USA 1996; 93:7387-7391.

22 Ishikawa Y, Kitamura M. Spontaneous apoptosis of podocytes in explanted glomeruli. Kidney Int 1998; 54:2008-2013.

23 Kitamura M. Adoptive transfer of NF- $\kappa$ B-inactive macrophages to the glomerulus. Kidney Int 2000;57: 709-716.

24 Ikezumi Y, Hurst LA, Masaki T, et al. Adoptive transfer studies demonstrate that macrophages can induce proteinuria and mesangial cell proliferation. Kidney Int 2003;63:83-95.

25 Kawachi H, Iwanaga T, Toyabe S, et al. Mesangial sclerotic change with persistent proteinuria in rats after two consecutive injections of monoclonal antibody 1-22-3. Clin Exp Immunol 1992;90:129-134.

26 Toyabe S, Iwanaga T. An ultrastructural study of proliferative nephritis induced experimentally by a monoclonal antibody against mesangial cells: replacement of mesangial cells by cells of the monocytemacrophage system. Virchows Archiv B Cell Pathol Incl Mol Pathol 1992;61:397-407.

27 Zoltick PW, Wilson JM. A quantitative nonimmunogenic transgene product for evaluating vectors in nonhuman primates. Mol Ther 2000;2:657-659. 